\title{
Systematic study of symmetry energy coefficient in finite nuclei
}

\author{
H. Mei ${ }^{1}$ Y. Huang, ${ }^{1,2}$ J. M. Yao, ${ }^{1}$ and H. Chen ${ }^{1}$ \\ ${ }^{1}$ School of Physical Science and Technology, \\ Southwest University, Chongqing 400715, China \\ ${ }^{2}$ School of Physics, Peking University, Beijing 100871, China
}

\begin{abstract}
The symmetry energy coefficients in finite nuclei have been studied systematically with a covariant density functional theory (DFT) and compared with the values calculated using several available mass tables. Due to the contamination of shell effect, the nuclear symmetry energy coefficients extracted from the binding energies have large fluctuations around the nuclei with double magic numbers. The size of this contamination is shown to be smaller for the nuclei with larger isospin value. After subtracting the shell effect with the Strutinsky method, the obtained nuclear symmetry energy coefficients with different isospin values are shown to decrease smoothly with the mass number $A$ and are subsequently fitted to the relation $\frac{4 a_{\mathrm{sym}}}{A}=\frac{b_{v}}{A}-\frac{b_{s}}{A^{4 / 3}}$. The resultant volume $b_{v}$ and surface $b_{s}$ coefficients from axially deformed covariant DFT calculations are 121.73 and $197.98 \mathrm{MeV}$ respectively. The ratio $b_{s} / b_{v}=1.63$ is in good agreement with the value derived from the previous calculations with the non-relativistic Skyrme energy functionals. The coefficients $b_{v}$ and $b_{s}$ corresponding to several available mass tables are also extracted. It is shown that there is a strong linear correlation between the volume $b_{v}$ and surface $b_{s}$ coefficients and the ratios $b_{s} / b_{v}$ are in between $1.6-2.0$ for all the cases.
\end{abstract}




\section{INTRODUCTION}

The advent of radioactive ion beams (RIBs) [1,2] provides a useful tool for studying exotic nuclei far from the $\beta$-stability. Hitherto, RIBs have already disclosed many structure phenomena in nuclei with extreme isospin values, and the next generation of radioactivebeam facilities are expected to produce more and more exotic nuclei [3-9]. These exotic nuclei provide us useful information on the equation of state (EOS) of asymmetric nuclear matter, which has not been well determined but is important for understanding both the structure of unstable nuclei and the properties of neutron stars. One of the most important quantities is the nuclear symmetry energy, which affects significantly the binding energy and radii of neutron-rich nuclei [10]. Furthermore, the chemical composition, the evolution of lepton profiles, cooling process and the neutrino fluxes in neutron stars depend strongly on the nuclear symmetry energy $[11,12]$.

Many investigations have already been carried out to study the density and isospin dependence of symmetry energy for nuclear matter [13-15]. The EOS for asymmetric nuclear

matter has a general parabolic form $\frac{E}{A}(\rho, \beta)=\frac{E}{A}(\rho)+E_{\mathrm{sym}}(\rho, 0) \beta^{2}+O\left(\beta^{2}\right)$ with baryon density $\rho=\rho_{n}+\rho_{p}$, isospin asymmetry $\beta=\left(\rho_{n}-\rho_{p}\right) / \rho$, and nuclear symmetry energy coefficient $E_{\mathrm{sym}}(\rho)=\left.\frac{1}{2 !} \frac{\partial^{2} E(\rho, \beta)}{\partial \beta^{2}}\right|_{\beta=0}$. Near the nuclear saturation density, the $E_{\mathrm{sym}}(\rho)$ has a strong influence on the neutron density distribution and thus on the neutron skin in stable and exotic nuclei. Previous studies have demonstrated that the neutron skin size can yield information on the derivative of symmetry energy with respect to density [16-21].

In recent years, the extraction of symmetry energy from finite nuclei has attracted much attention. According to the semi-empirical mass formula of liquid drop model (LDM), the symmetry energy in finite nuclei is proportional to the square of the difference between neutron and proton numbers. However, recent studies indicate that the symmetry energy is more reasonable to be parameterized as $E_{\text {sym }} \sim T(T+c)$ with $T=|N-Z| / 2[22]$. The empirical fits in Ref. [23] show a clear preference for $c=1$. The fits with either $c=0$ as in the LDM, or with $c=4$ as in the Wigner SU(4) symmetry are disfavored. Similar conclusion has also been reached in Ref. [24]. Later on, the symmetry energy of finint nuclei has been examined with several microscopic models, including the Hartree-Bogoliubov approach with random phase approximation [25], and energy density functional (EDF) of Skyrme force [26] or the relativistic meson-exchange interaction [27], all of which favor $c=1$ as well. 
On the other hand, it has been shown that the surface tension needs to depend on asymmetry. This dependence modifies the surface energy and implies the emergence of asymmetry skin [13]. It was pointed out that the surface symmetry term was required in the symmetry energy coefficient, namely, $\frac{4 a_{\mathrm{sym}}}{A}=\frac{b_{v}}{A}-\frac{b_{s}}{A^{4 / 3}}$, in order to describe the energies of light asymmetric nuclei at the level similar to the other nuclei. Its physical origin is traditionally explained in terms of the kinetic energy and mean isovector potential contributions respectively [28]. In the previous studies, the ratio of the surface-to-volume contributions to the symmetry energy coefficient $r_{S / V}\left(\equiv b_{s} / b_{v}\right)$ has been estimated from the electric dipole strength distribution using the hydrodynamical model [29], or from the experimental masses [30-33], mass and radii [13, 34], or the excitation energies of isobaric analog states [35]. Recently, an attempt has also been made to extract symmetry energy and the ratio $r_{S / V}$ from the separation energies through the displacement of neutron and proton chemical potentials [36].

The density functional theory (DFT) in nuclear physics is nowadays the most important microscopic approach for large-scale nuclear structure calculations in medium-heavy and heavy nuclei and it has been successfully employed for the description of nuclei around and far from the $\beta$-stability [37]. In the framework of DFT [38], the LDM parameters and effective symmetry energy coefficient were extracted for nuclei with huge numbers of nucleons, of the order of $10^{6}$. In the mean time, the systematical extraction of symmetry energy coefficient from realistic finite nuclei, for which, the surface energy term is important, was carried out based on several non-relativistic Skyrme EDFs [39], where the global mass dependence of symmetry energy coefficient was studied by switching off the Coulomb and pairing effects. It has been found that the ratio of the surface-to-volume contributions to the symmetry energy coefficient, $r_{S / V}\left(\equiv b_{s} / b_{v}\right)$ is around 1.6, which is consistent with the value of Ref. [32], but much different from the estimation $2.0 \leq r_{S / V} \leq 2.8$ in Ref. [13]. However, the recent careful study with the considerations of Coulomb interaction and shell effect in Ref. [40] shows that this ratio could be quite different for different Skyrme forces, for instance, $r_{S / V}=1.2$ for BSk6, 1.7 for SkM*, and 2.2 for SkI3.

In the past decades, nuclear covariant DFT has achieved comparable success with the nonrelativistic DFT in the description of ground state properties of both spherical and deformed nuclei all over the nuclear chart [41-44]. In particular, there are many advantages using the EDF with manifest covariance, including natural inclusion of the nucleon spin degree of 
freedom, automatical emergence of nuclear spin-orbit potential, an unique parametrization of time-odd components (nuclear currents and magnetism) in nuclear mean-field as well as the natural saturation mechanism for nuclear matter.

In recent several years, the covariant DFT theory with point-coupling interaction has attracted much more attention. It shows great advantages in the extension for nuclear lowlying excited states by using projection techniques [45], generator coordinate methods [46-49] and collective Hamiltonian [50]. In this framework, the PC-PK1 set [51] was proposed most recently by fitting to the binding energies of 60 selected spherical nuclei and the charge radii of 17 selected spherical nuclei from $\mathrm{O}$ to $\mathrm{Pb}$ isotopes. The success of PC-PK1 has been illustrated in the description of infinite nuclear matter and finite nuclei for both groundstate and low-lying excited states. Furthermore, the PC-PK1 provides a good description for the isospin dependence of nuclear binding energy along either isotopic or isotonic chain, which is particular important for description of nuclear symmetry energy in finite nuclei. In view of these facts, in this paper, we will study the symmetry energy coefficient of realistic finite nuclei systematically using both spherical and axially deformed RMF approaches with the PC-PK1 covariant EDF, where the shell correction energy will be subtracted using the Strutinsky method [52]. The obtained symmetry energy coefficient will be compared with those extracted from the binding energies of several available mass tables. Compared with our previous work with the PK1 effective interaction [53], in this work, we make a lot of improvements, including the consideration of shell correction energy, axial deformation, pairing correlation and Coulomb interaction in the RMF calculations, which might have influences on the extracted symmetry energy of realistic nuclei.

The paper is organized as follows. In Sec. II we briefly describe the method used to extract the nuclear symmetry coefficient in finite nuclei. The results and discussion will be given in Sec. III. Finally, a summary is presented in Sec. IV.

\section{THE METHOD}

\section{A. The relativistic mean-field approach with point-coupling interaction}

The relativistic mean-field (RMF) approach with point-coupling interaction for nucleons has been described in detail in Refs. $[45,51]$. Here, we present only the outline of this 
approach. It starts from the following Lagrangian density,

$$
\begin{aligned}
\mathcal{L}= & \bar{\psi}\left(i \gamma_{\mu} \partial^{\mu}-m\right) \psi \\
& -\frac{1}{2} \alpha_{S}(\bar{\psi} \psi)(\bar{\psi} \psi)-\frac{1}{2} \alpha_{V}\left(\bar{\psi} \gamma_{\mu} \psi\right)\left(\bar{\psi} \gamma^{\mu} \psi\right) \\
& \left.-\frac{1}{2} \alpha_{T V}\left(\bar{\psi} \vec{\tau} \gamma_{\mu} \psi\right)\left(\bar{\psi} \vec{\tau} \gamma^{\mu} \psi\right)\right) \\
& -\frac{1}{2} \delta_{S} \partial_{\nu}(\bar{\psi} \psi) \partial^{\nu}(\bar{\psi} \psi)-\frac{1}{2} \delta_{V} \partial_{\nu}\left(\bar{\psi} \gamma_{\mu} \psi\right) \partial^{\nu}\left(\bar{\psi} \gamma^{\mu} \psi\right) \\
& -\frac{1}{2} \delta_{T V} \partial_{\nu}\left(\bar{\psi} \vec{\tau} \gamma_{\mu} \psi\right) \partial^{\nu}\left(\bar{\psi} \vec{\tau} \gamma^{\mu} \psi\right) \\
& -\frac{1}{3} \beta_{S}(\bar{\psi} \psi)^{3}-\frac{1}{4} \gamma_{S}(\bar{\psi} \psi)^{4}-\frac{1}{4} \gamma_{V}\left[\left(\bar{\psi} \gamma_{\mu} \psi\right)\left(\bar{\psi} \gamma^{\mu} \psi\right)\right]^{2} \\
& -\frac{1}{4} F^{\mu \nu} F_{\mu \nu}-e \bar{\psi} \gamma^{\mu} \frac{1-\tau_{3}}{2} \psi A_{\mu},
\end{aligned}
$$

which contains 9 coupling constants $\alpha_{S}, \alpha_{V}, \alpha_{T V}, \beta_{S}, \gamma_{S}, \gamma_{V}, \delta_{S}, \delta_{V}$ and $\delta_{T V}$. The subscripts indicate the symmetry of the couplings: $S$ stands for scalar, $V$ for vector, and $T$ for isovector, while the symbol refer to the additional distinctions: $\alpha$ refers to four-fermion term, $\delta$ to derivative couplings, and $\beta$ and $\gamma$ to the third- and fourth-order terms, respectively.

Using the mean-field approximation and the "no-sea" approximation, one finds the energy density functional corresponding to the Lagrangian density (1). Minimization of the energy density functional with respect to $\bar{\psi}_{k}$ gives rise to the Dirac equation (i.e., Kohn-Sham equation) for the single nucleon wave function $\psi_{k}$,

$$
\left[\gamma_{\mu}\left(i \partial^{\mu}-V^{\mu}\right)-(m+S)\right] \psi_{k}=0
$$

The single-particle effective Hamiltonian contains local scalar $S(\boldsymbol{r})$ and vector $V^{\mu}(\boldsymbol{r})$ potentials

$$
S(\boldsymbol{r})=\Sigma_{S}, \quad V^{\mu}(\boldsymbol{r})=\Sigma^{\mu}+\vec{\tau} \cdot \vec{\Sigma}_{T V}^{\mu}
$$

where the nucleon scalar-isoscalar $\Sigma_{S}$, vector-isoscalar $\Sigma^{\mu}$ and vector-isovector $\vec{\Sigma}_{T V}^{\mu}$ selfenergies are given in terms of the various densities and currents,

$$
\begin{aligned}
\Sigma_{S} & =\alpha_{S} \rho_{S}+\beta_{S} \rho_{S}^{2}+\gamma_{S} \rho_{S}^{3}+\delta_{S} \triangle \rho_{S}, \\
\Sigma^{\mu} & =\alpha_{V} j_{V}^{\mu}+\gamma_{V}\left(j_{V}^{\mu}\right)^{3}+\delta_{V} \triangle j_{V}^{\mu}+e A^{\mu}, \\
\vec{\Sigma}_{T V}^{\mu} & =\alpha_{T V} \vec{j}_{T V}^{\mu}+\delta_{T V} \triangle \vec{j}_{T V}^{\mu} .
\end{aligned}
$$


The local densities and currents are defined by,

$$
\begin{aligned}
\rho_{S}(\boldsymbol{r}) & =\sum_{k} v_{k}^{2} \bar{\psi}_{k}(\boldsymbol{r}) \psi_{k}(\boldsymbol{r}), \\
j_{V}^{\mu}(\boldsymbol{r}) & =\sum_{k} v_{k}^{2} \bar{\psi}_{k}(\boldsymbol{r}) \gamma^{\mu} \psi_{k}(\boldsymbol{r}), \\
\vec{j}_{T V}^{\mu}(\boldsymbol{r}) & =\sum_{k} v_{k}^{2} \bar{\psi}_{k}(\boldsymbol{r}) \vec{\tau} \gamma^{\mu} \psi_{k}(\boldsymbol{r}),
\end{aligned}
$$

where the summation $\sum_{k}$ runs over only positive-energy states with the occupation probabilities $v_{k}^{2}$.

For ground state of an even-even nucleus one has time reversal symmetry. The space-like components of the currents and the spatial part of the vector potential vanish. Moreover, because of charge conservation in nuclei, only the 3rd-component of isovector potential $\vec{\Sigma}_{T V}^{\mu}$ contributes. The Coulomb field $A_{0}$ is determined by Poisson's equation.

Pairing correlations between nucleons are treated in the BCS approximation, where the density-independent $\delta$-force is used in the particle-particle channel. Therefore, the nuclear total energy is contributed from both particle-hole and particle-particle channels. Moreover, the center-of-mass (c.m.) correction to the total energy is taken into account microscopically,

$$
E_{\mathrm{cm}}^{\mathrm{mic}}=-\frac{1}{2 m A}\left\langle\hat{P}_{\mathrm{cm}}^{2}\right\rangle
$$

where $m$ is the mass of nucleon, and $A$ is mass number. $\hat{P}_{\mathrm{cm}}=\sum_{i}^{A} \hat{p}_{i}$ is the total momentum in the c.m. frame.

\section{B. Extraction of symmetry energy coefficient}

In the conventional semi-empirical mass formula of LDM for the binding energy of nuclei, the symmetry energy is given in terms of the isospin value $T$ [52],

$$
E_{\mathrm{sym}}(A, T)=\frac{4 a_{\mathrm{sym}}}{A}(A, T) T^{2},
$$

where $A$ is the mass number. $\frac{4 a_{\text {sym }}}{A}(A, T)$ represents the symmetry energy coefficient. The isospin value $T$ is given by $|T|=|N-Z| / 2$. Recent microscopic studies [23, 25-27, 54] with the consideration of nuclear Wigner energy showed that the nuclear symmetry energy is more proper to relate the isospin value $T$ by the following relation,

$$
E_{\mathrm{sym}}(A, T)=\frac{4 a_{\mathrm{sym}}}{A}(A, T) T(T+1)
$$


On the other hand, according to the mass formula of LDM, the difference between binding energies of isobaric nuclei with the same odd-even parity is only related to the Coulomb energy and symmetry energy terms,

$$
B(A, T)-B(A, 0)=E_{\text {Coul. }}(A, T)-E_{\text {Coul. }}(A, 0)+E_{\text {sym }}(A, T)-E_{\text {sym }}(A, 0),
$$

where $B(A, 0)$ is the total binding energy of $N=Z$ isobaric nucleus with the mass number A. Subsequently, the nuclear symmetry energy in Eq.(8) is simply determined by subtracting the contribution of Coulomb energy from the nuclear total binding energy as,

$$
E_{\text {sym }}(A, T)=[B(A, T)-B(A, 0)]-\left[E_{\text {Coul. }}(A, T)-E_{\text {Coul. }}(A, 0)\right] .
$$

Combining Eqs.(8) and (10), one can determine the value of symmetry energy coefficient if one knows the total binding energy and Coulomb energy of the concerned nucleus. The shell correction energy can contaminate the symmetry energy obtained in this way and therefore should be subtracted from the binding energy.

\section{RESULTS AND DISCUSSION}

In the RMF calculations, we adopt the recent parameterized relativistic point-coupling interaction PC-PK1 [51], which has been adjusted to the binding energies and charge radii of spherical nuclei from $\mathrm{O}$ to $\mathrm{Pb}$ isotopes. With the restriction of spherical/axial symmetry, the Dirac equation for nucleons is solved by expanding the Dirac spinor $\psi_{k}$ on a set of harmonic oscillator basis with 20/16 shells. Pairing correlations between nucleons are treated with the BCS approximation using a density-independent $\delta$ force. The pairing strengths have been adjusted to fit the average neutron pairing gaps in ${ }^{122} \mathrm{Sn},{ }^{124} \mathrm{Sn}{ }^{200} \mathrm{~Pb}$ and the average

proton gaps in ${ }^{92} \mathrm{Mo},{ }^{136} \mathrm{Xe}$, and ${ }^{144} \mathrm{Sm}$. More details about the numerical calculations can be found in Ref. [51].

Figure 1 displays the nuclear symmetry energy coefficient for finite nuclei with $8 \leq Z \leq 70$ and $T=2,4, \cdots, 12$ from the spherical RMF calculations with effective interaction PCPK1 [51] as a function of the corresponding mass number $A$. The filled (open) symbols represent the values with (without) taking into account the shell correction energies that are calculated with the Strutinsky method [52]. It shows clearly that due to the contamination of shell correction energy, the resultant nuclear symmetry energy coefficients have 


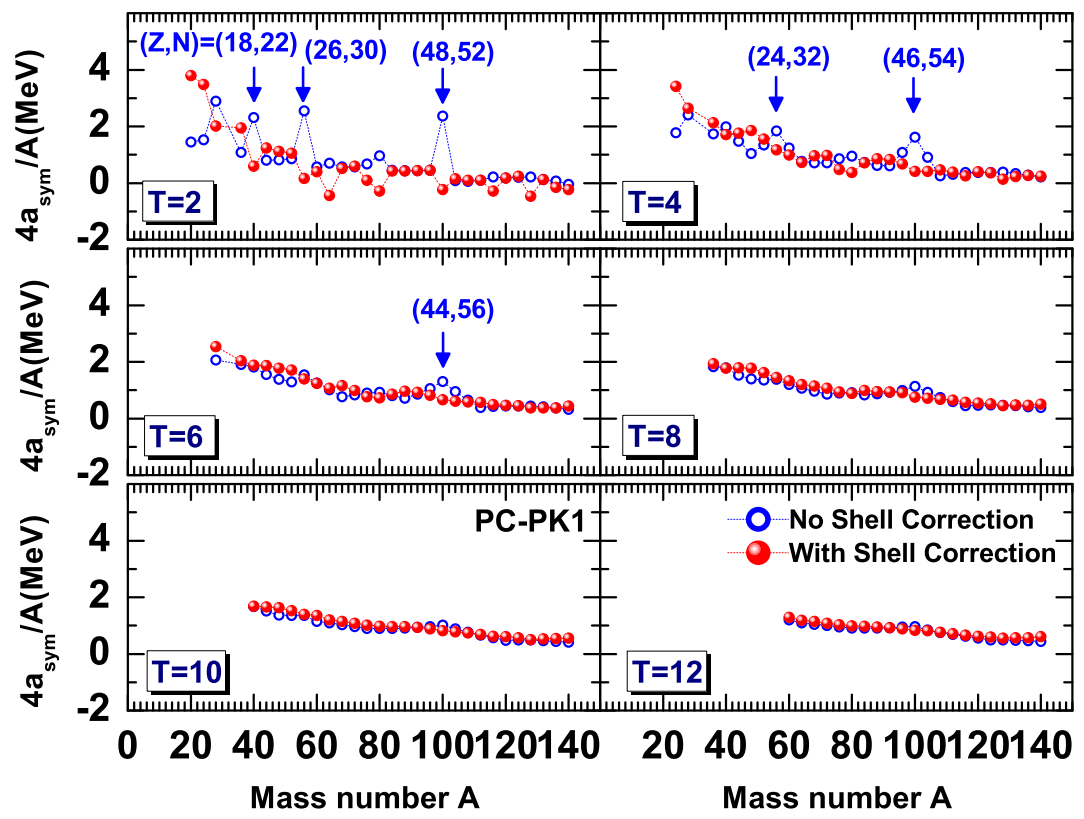

FIG. 1: The nuclear symmetry energy coefficients for finite nuclei with $8 \leq Z \leq 70$ and $T=$ $2,4, \cdots, 12$ in the spherical relativistic mean-field calculations with effective interaction PC-PK1 as functions of the corresponding mass number $A$. The filled (open) symbols represent the values with (without) taking into account the shell correction.

large fluctuations around the nuclei with double magic numbers. Moreover, the amplitude of these fluctuations decreases with the isospin value $T$. It indicates that the symmetry energy becomes less sensitive to the shell effect for the nuclei with larger isospin value $T$. After subtracting the shell effect energy evaluated with the Strutinsky method, the obtained nuclear symmetry energy coefficients are smoothly decreasing with the mass number $A$.

Furthermore, the nuclear symmetry energy coefficients are calculated using the binding energy of nuclei with $T=2,4, \cdots, 10$ from several available mass tables, including the "DZ28" [54], "FRDM" [32], "HFB17" [55], "RMF(TMA)" [56], "ETFSIQ" [57] as well as the experimental data "Audi03" [58]. In the calculations, we take the Coulomb energy $E_{\text {Coul. }}(A, T)$ of a specific nucleus as the value of an uniformly charged droplet, i.e.,

$$
E_{\text {Coul. }}(A, T)=\frac{3}{5} \frac{Z^{2} e^{2}}{R_{c}}\left[1-\frac{5}{4}\left(\frac{3}{2 \pi}\right)^{2 / 3} \frac{1}{Z^{2 / 3}}\right], \quad R_{c}=1.2 A^{1 / 3} .
$$

Figure 2 shows the resultant symmetry energy coefficient from different mass tables as a function of the mass number $A$. Since the shell effect is not subtracted from the binding 


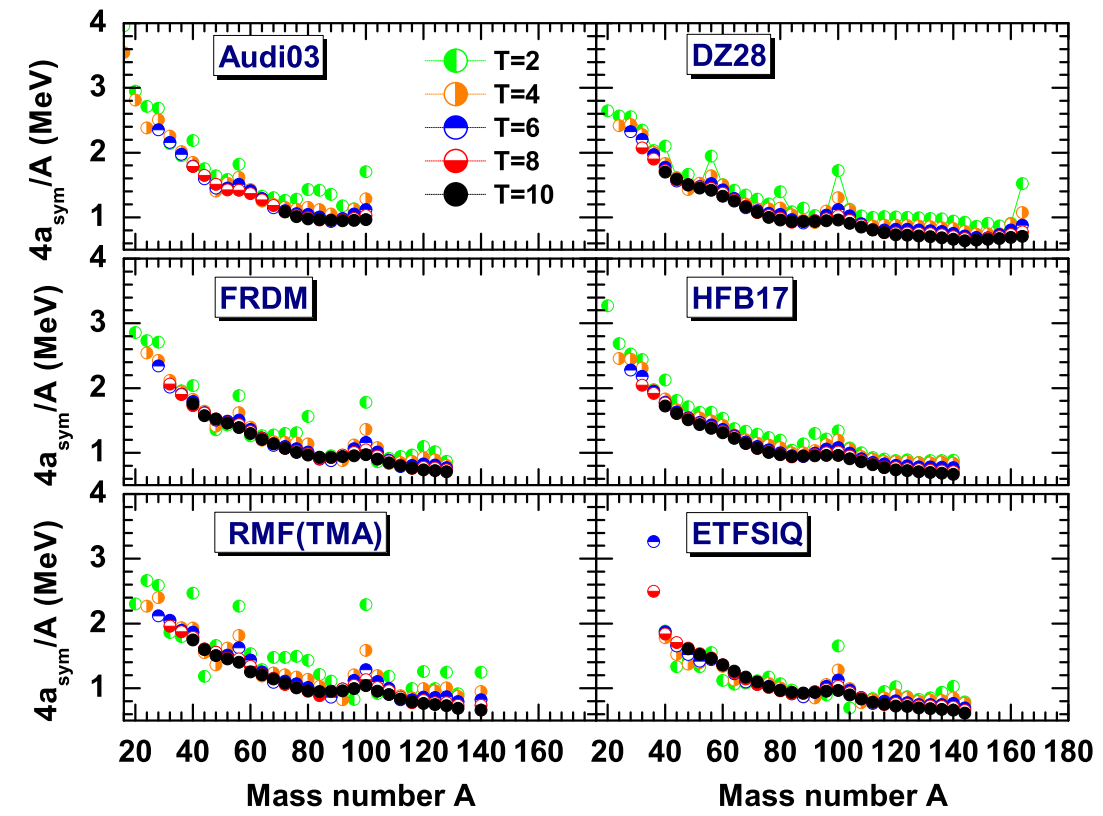

FIG. 2: The nuclear symmetry energy coefficient for finite nuclei with $T=2,4, \cdots, 10$ respectively in different mass tables as a function of the corresponding mass number $A$.

energies of these mass tables, similar fluctuation as those in Fig. 1 (open symbols) is observed in Fig. 2.

The symmetry energy coefficient of finite nucleus with mass number $A$ is usually parameterized as follows,

$$
\frac{4 a_{\mathrm{sym}}}{A}=\frac{b_{v}}{A}-\frac{b_{s}}{A^{4 / 3}}
$$

In Ref. [54], it was found that $b_{v}=134.4, b_{s}=203.6 \mathrm{MeV}$, while in Ref. [27], the axial RMF calculations with the PK1 [59] interaction for the $A=40,48,56,88,100,120,140,160,164$, and 180 isobaric chains gave $b_{v}=133.2$ and $b_{s}=220.3 \mathrm{MeV}$. We note that in Ref. [27], the Coulomb interaction between protons was switched off and the contamination of shell effect was not considered in the calculations. Since the shell effect in nuclear symmetry energy coefficient is much weaker for high isospin value as shown in Fig. 1 and Fig. 2, we perform a global fit of the symmetry energy coefficients to the relation (12) via the LevenbergMarquardt method. Considering the limited experimental data for nuclei with $T=10$, the corresponding experimental value "Audi03" extracted from the finite nuclei with $T=8$ is also shown in Fig. 3. The obtained $b_{v}$ and $b_{s}$ values, together with the standard root-mean- 


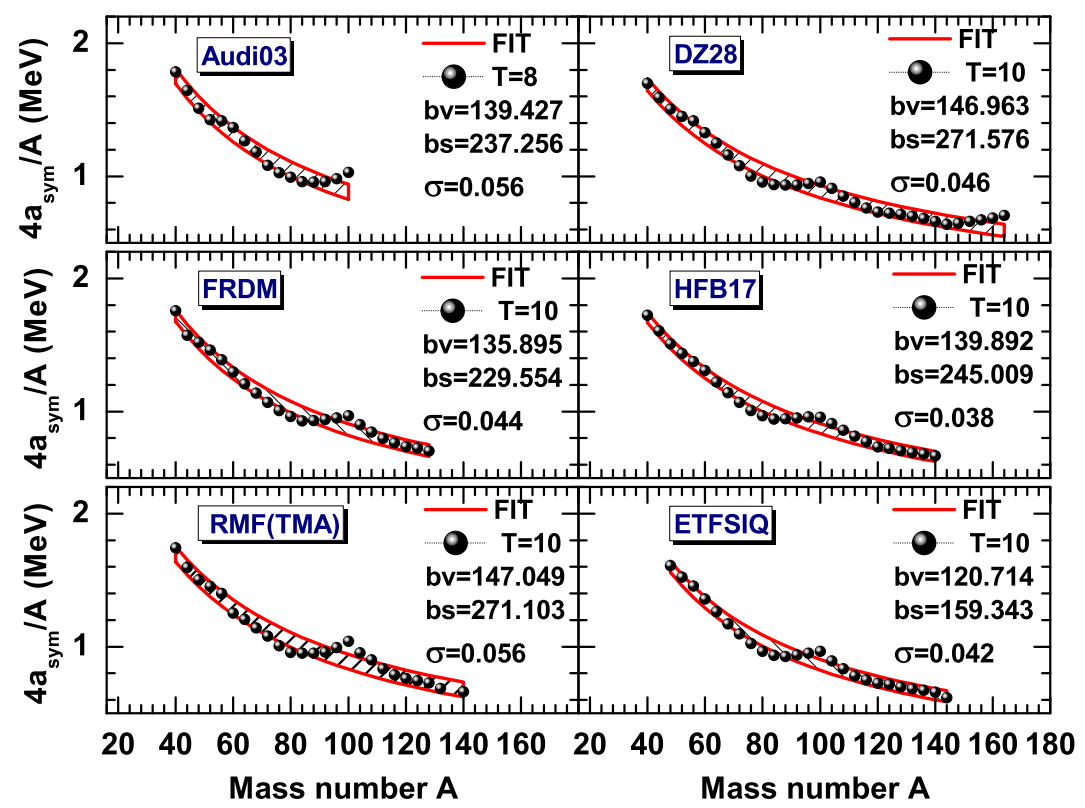

FIG. 3: The nuclear symmetry energy coefficient extracted from finite nuclei with $T=10$ in different mass tables. The optimal values of volume coefficient $b_{v}$ and surface coefficient $b_{s}$ fitted to the relation (12) and the corresponding standard root-mean-square errors $\sigma$ are presented. The shade area indicates the $\frac{4 a_{\mathrm{sym}}}{A} \pm 1 \sigma$, where $\frac{4 a_{\mathrm{sym}}}{A}$ is calculated using the corresponding optimal values of $b_{v}$ and $b_{s}$.

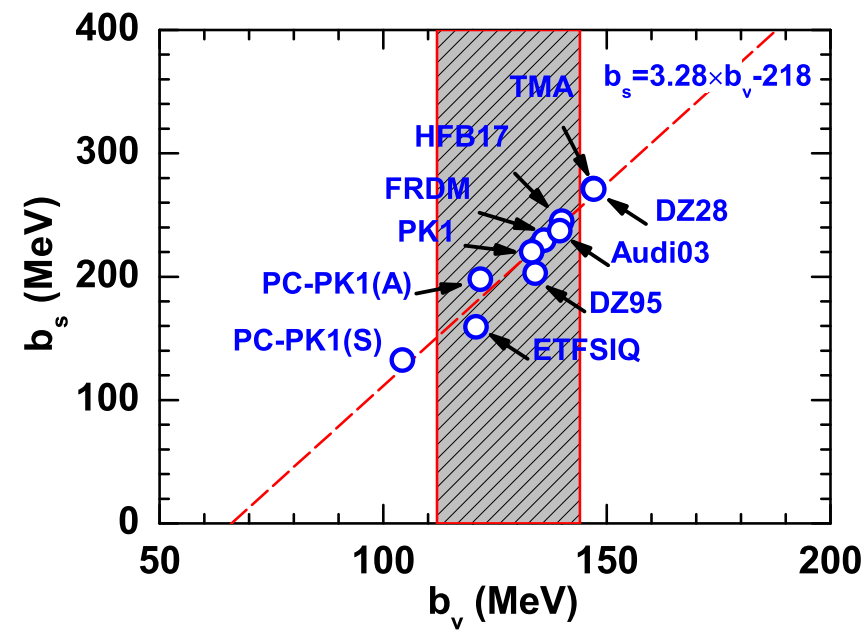

FIG. 4: The surface coefficient $b_{s}$ as a function of the volume coefficient $b_{v}$. The shade area indicates the empirical value of $b_{v}=128 \pm 16(\mathrm{MeV})$ for infinite nuclear matter (see text for details). 
square errors $\sigma$ are presented. The small $\sigma$ value indicates the good quality of the fits. The resultant volume coefficient $b_{v}$ and surface coefficient $b_{s}$ are plotted in Fig. 4, where the shade area indicates the empirical value of $b_{v}=128 \pm 16 \mathrm{MeV}$ (corresponding to $a_{\text {sym }}=32 \pm 4$ $\mathrm{MeV}$ for infinite nuclear matter). In addition, the values of $b_{v}$ and $b_{s}$ from the axial RMF calculations with the PK1 [59] effective interactions (labeled as "PK1") from Ref. [27], as well as the spherical/axial RMF calculations with the PC-PK1 effective interaction (labeled as "PC-PK1(S)/PC-PK1(A)") from this work are plotted for comparison. It is shown that all the obtained $b_{v}$ values are in good agreement with the empirical value, except the case of spherical RMF calculations with the PC-PK1 interaction, which gives $b_{v}=104.25$ and $b_{s}=132.38 \mathrm{MeV}$. After taking into account the effect of axial deformation, the obtained $b_{v}$ value of "PC-PK1(A)" agrees well with the empirical value, with $b_{v}=121.73$ and $b_{s}=197.98$ $\mathrm{MeV}$ respectively. The ratio $r_{S / V}=1.63$ is in good agreement with the value derived from the previous calculations with the non-relativistic Skyrme energy functionals [39]. Furthermore, it is shown in Fig. 4 that there is a strong linear correlation (by the relation $b_{s}=3.28 b_{v}-218$ $\mathrm{MeV}$ ) between the volume $b_{v}$ and surface $b_{s}$ coefficients in the symmetry energy coefficient of finite nucleus, which is consistent with the results in Ref. [34].

Furthermore, we fit the values of $b_{v}$ and $b_{s}$ to the symmetry energy coefficients using the binding energies of nuclei in different mass tables corresponding to smaller values of isospin, i.e., $T=6$ and 8 . Figure 5 displays the obtained $b_{v}$ and $b_{s}$ as functions of the isospin value. It is shown that except the "ETFSIQ", the obtained $b_{s}$ and $b_{v}$ change slowly with the isospin value and have the tendency to be convergent at $T=10$, which is consistent with the observation in Fig. 2.

In Fig. 6, we plot the surface coefficient $b_{s}$ and the surface-to-volume ratio $r_{S / V}$ extracted from the binding energies of finite nuclei with $T=6,8,10$ as functions of the volume coefficient $b_{v}$. It is shown that all the $b_{s}$ and $b_{v}$ are linearly correlated. The ratios $r_{S / V}$ of all cases, except "ETFSIQ", are in between 1.6 and 2.0, which is consistent with the values from the hydrodynamical model calculation [29], the Skryme-Hartree-Fock (SHF) model calculation in Ref. [39] as well as the result $r_{S / V} \simeq 1.7$ derived from the shift of neutron-proton chemical potentials for nuclei beyond the $\beta$-stability line with $A \geq 50$ [36]. 


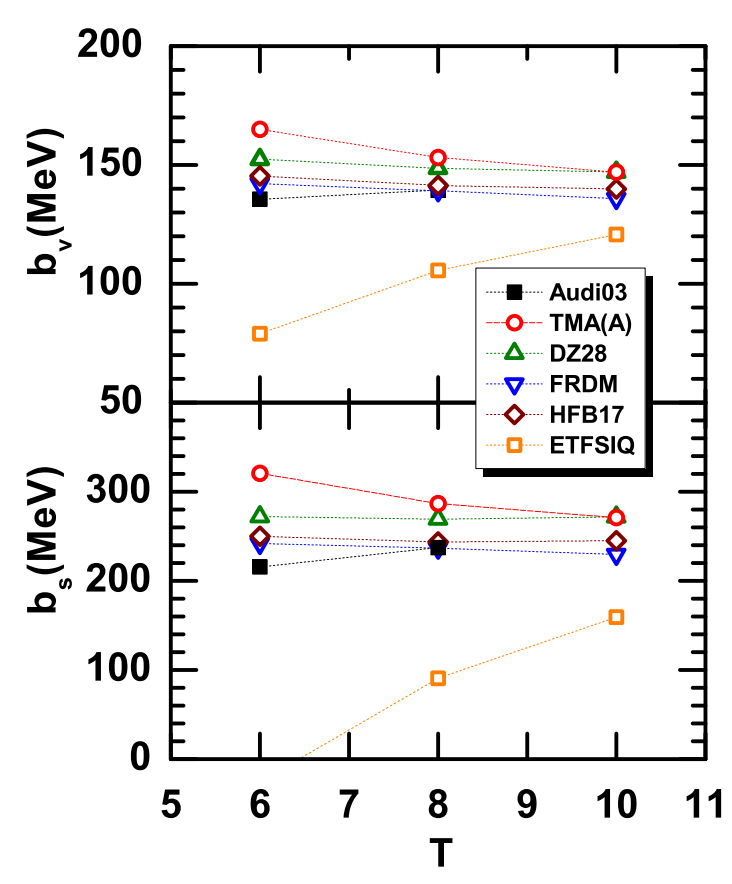

FIG. 5: The optimal values of volume $b_{v}$ and surface $b_{s}$ coefficient, fitted to the relation (12) of nuclear symmetry energy coefficient for finite nuclei with $T=6,8,10$ in different mass tables.

\section{SUMMARY}

The symmetry energy coefficient in finite nuclei have been studied systematically in the RMF approach with the point-coupling effective interaction PC-PK1 and compared with the values calculated using the several available mass tables. Due to the contamination of shell effect, the resultant nuclear symmetry energy coefficients have large fluctuations around the nuclei with double magic numbers. This shell effect in symmetry energy coefficient is less important for the nuclei with larger isospin value. After subtracting the shell effect with the Strutinsky method, the obtained nuclear symmetry energy coefficients have been shown to decrease smoothly with the mass number $A$. Moreover, the symmetry energy coefficients have been fitted to the relation $\frac{4 a_{\text {sym }}}{A}=\frac{b_{v}}{A}-\frac{b_{s}}{A^{4 / 3}}$. The resultant volume $b_{v}$ and surface $b_{s}$ coefficients from axially deformed calculations are 121.73 and $197.98 \mathrm{MeV}$ respectively. The ratio $r_{S / V}=1.63$ is in good agreement with the value derived from the previous calculations with the non-relativistic Skyrme energy functionals in Ref. [39]. The coefficients $b_{v}$ and $b_{s}$ corresponding to several available mass tables have also been extracted. It has been shown 


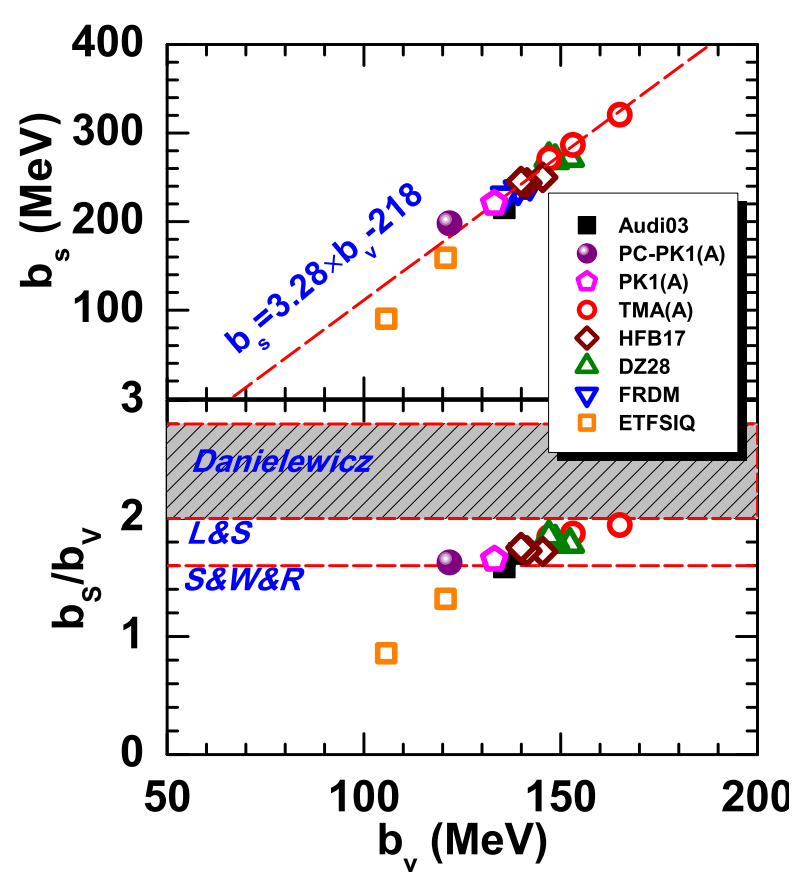

FIG. 6: The surface coefficient $b_{s}$ and the surface-to-volume ratio $r_{S / V}\left(\equiv b_{s} / b_{v}\right)$ extracted from the binding energies of finite nuclei with $T=6,8,10$ in different mass tables as functions of the volume coefficient $b_{v}$. The results of axial RMF calculations with the PC-PK1 and PK1 interactions are given as well. The dashed lines indicates the values from the hydrodynamical model calculation [29] and the SHF model calculation [39]. The shade area indicates the estimation in Ref. [13].

that there is a strong linear correlation between the volume $b_{v}$ and surface $b_{s}$ coefficients, which is consistent with the results of previous study [34]. Moreover, the ratios $r_{S / V}$ have been shown in between $1.6-2.0$ for all the cases.

\section{Acknowledgments}

We would like to thank S. F. Ban, J. Meng, and W. Satula for fruitful discussions and thank Z. P. Li, P. W. Zhao and Q. B. Chen for critical reading of this manuscript. J.M.Y. acknowledges a postdoctoral fellowship from the F.R.S.-FNRS (Belgium). This work is partly supported by the National Natural Science Foundation of China under Grant No. 11105111 and No. 10947013, the Fundamental Research Funds for the Central Universities (XDJK2010B007) and the Southwest University Initial Research Foundation Grant to 
Doctor under Grant No. SWU109011.

[1] I. Tanihata, H. Hamagaki, O. Hashimoto et al., Phys. Rev. Lett. 55, 2676 (1985).

[2] C. Bertulani, M. Hussein, and G. Münzenberg, Physics of Radioactive Beams (Nova Science Publishers Inc., 2001).

[3] A. Mueller and B. Sherrill, Ann. Rev. Nucl. Part. Sci. 43, 529 (1993).

[4] I. Tanihata, Prog. Part. Nucl. Phys. 35, 505 (1995).

[5] P. Hansen, A. S. Jensen, and B. Jonson, Annu. Rev. Nucl. Part. Phys. 45, 591 (1995).

[6] R. F. Casten and B. M. Sherrill, Prog. Part. Nucl. Phys. 45, S171 (2000).

[7] A. Mueller, Prog. Part. Nucl. Phys. 48, 359 (2001).

[8] B. Jonson, Phys. Rep. 389, 1 (2004).

[9] A. Jensen, K. Riisager, D. Fedorov, and E. Garrido, Rev. Mod. Phys. 76, 215 (2004).

[10] K. Sumiyoshi, D. Hirata, H. Toki, and H. Sagawa, Nucl. Phys. A552, 437 (1993).

[11] K. Sumiyoshi, H. Suzuki, and H. Toki, Astron. Astrophys. 303, 475 (1995).

[12] R. R. Chasman, Phys. Lett. B577, 47 (2003).

[13] P. Danielewicz, Nucl. Phys. A727, 233 (2003).

[14] V. Baran, M. Colonna, V. Greco, M. Di Toro, Phys. Rep. 410, 335 (2005).

[15] B. A. Li, L. W. Chen and C. M. Ko, Phys. Rep. 464, 113 (2008) and references therein.

[16] K. Oyamatsu, I. Tanihata, Y. Sugahara, K. Sumiyoshi, H. Toki, Nucl. Phys. A634, 3 (1998).

[17] B. A. Brown, Phys. Rev. Lett. 85, 5296 (2000).

[18] S. Typel, B. A. Brown, Phys. Rev. C64, 027302 (2001).

[19] R. J. Furnstahl, Nucl. Phys. A706, 85 (2002).

[20] M. Centelles, X. Roca-Maza X. Viñas, and M. Warda, Phys. Rev. Lett. 102, 122502 (2009).

[21] X. Roca-Maza, M. Centelles, X. Viñas, and M. Warda, Phys. Rev. Lett. 106, 252501 (2011).

[22] P. Vogel, Nucl. Phys. A662, 148 (2000).

[23] J. Jänecke, Nucl. Phys. 73, 97 (1965); J. Jänecke et al., Nucl. Phys. A728, 23 (2003).

[24] A. S. Jensen, P. G. Hansen, B. Jonson, Nucl. Phys. A431, 393 (1984).

[25] K. Neergard, Phys. Lett. B537, 287 (2002); Phys. Lett. B572, 159 (2003).

[26] W. Satula and R. A. Wyss, Phys. Lett. B572, 152 (2003).

[27] S. F. Ban, J. Meng, W. Satula, and R. A. Wyss, Phys. Lett. B633, 231 (2006). 
[28] A. Bohr and B. R. Mottelson, Nuclear Structure, Vol. I (Benjamin, New York, 1969).

[29] E. Lipparini, S. Stringari, Phys. Lett. B112, 421 (1982).

[30] T. Mukhopadhyay and D. N. Basu, Nucl. Phys. A789, 201 (2007).

[31] M. W. Kirson, Nucl. Phys. A798, 29 (2008).

[32] P. Moller, J. R. Nix, W. D. Myers et al., Atom. Data Nucl. Data Tables 59, 185 (1995).

[33] N. Wang and M. Liu, Phys. Rev. C81, 067302 (2010).

[34] A. E. L. Dieperink and P. Van Isacker, Eur. Phys. J. A32, 11 (2007).

[35] P. Danielewicz and J. Lee, AIP Conf. Proc. 947, 301 (2007).

[36] V. M. Kolomietz and A. I. Sanzhur, Phys. Rev. C81, 024324 (2010).

[37] M. Bender, P.-H. Heenen, and P.-G. Reinhard, Rev. Mod. Phys. 75, 121 (2003).

[38] P.-G. Reinhard, M. Bender, W. Nazarewicz, and T. Vertse, Phys. Rev. C73, 014309 (2006).

[39] W. Satula, R. A. Wyss and M. Rafalski, Phys. Rev. C74, 011301(R) (2006).

[40] N. Nikolov, N. Schunck, W. Nazarewicz et al., Phys. Rev. C83, 034305 (2011).

[41] P. G. Reinhard, Rep. Prog. Phys. 52, 439 (1989).

[42] P. Ring, Prog. Part. Nucl. Phys. 37, 193 (1996).

[43] D. Vretenar et al., Phys. Rep. 409, 101 (2005).

[44] J. Meng et al., Prog. Part. Nucl. Phys. 57, 470 (2006).

[45] J. M. Yao, J. Meng, P. Ring, and D. Pena Arteaga, Phys. Rev. C79, 044312 (2009).

[46] T. Nikšić, D. Vretenar, and P. Ring, Phys. Rev. C74, 064309 (2006).

[47] J. M. Yao, J. Meng, P. Ring, and D. Vretenar, Phys. Rev. C81, 044311 (2010).

[48] J. M. Yao, H. Mei, H. Chen et al., Phys. Rev. C83, 014308 (2011).

[49] J. M. Yao, J. Meng, P. Ring et al., Phys. Rev. C84, 024306 (2011).

[50] T. Niksic, Z. P. Li, D. Vretenar et al., Phys. Rev. C79, 034303 (2009).

[51] P. W. Zhao, Z. P. Li, J. M. Yao, and J. Meng, Phys. Rev. C82, 054319 (2010).

[52] P. Ring and P. Schuck, The Nuclear Many-Body Problem (Springer, Heidelberg, 1980).

[53] H. Mei, J. M. Yao, and H. Chen, arXiv:0911.1204v1 [nucl-th].

[54] J. Duflo and A.P. Zuker, Phys. Rev. C52, R23 (1995).

[55] S. Goriely, N. Chamel, and J. M. Pearson, Phys. Rev. Lett. 102, 152503 (2009)

[56] L. S. Geng, H. Toki, and J. Meng, Prog. Theor. Phys. 113, 785 (2005).

[57] J. M. Pearson, R. C. Nayak, and S. Goriely, Phys. Lett. B387, 455 (1996).

[58] G. Audi, A. H. Wapstra, and C. Thibault, Nucl. Phys. A729, 337 (2003). 
[59] W. H. Long, J. Meng, N. V. Giai, and S. G. Zhou, Phys. Rev. C69, 034319 (2004). 